\title{
Models Underpinning Mental Health: A Proposal for A User-Based Framework
}

\author{
Joanna Fox* \\ Faculty of Health \& Education, Medicine and Social Care, Anglia Ruskin University, UK \\ *Corresponding author: Joanna Fox, Faculty of Health, Education, Medicine and Social Care, Anglia Ruskin University, UK. \\ To Cite This Article: Joanna Fox. Models Underpinning Mental Health: A Proposal for A User-Based Framework. Am J Biomed Sci \& Res. 2021 - \\ 13(3). AJBSR.MS.ID.001863. DOI: 10.34297/AJBSR.2021.13.001863.
}

Received: 眥 June 11, 2021; Published: 眥 June 23, 2021

\begin{abstract}
Purpose: This article seeks to explore the models of mental health used in the delivery of care and the impact theoretical frameworks have on the implementation of practice. It discusses the development of a user-led model of care which is based on forms of practice which enhance the place of experiential knowledge in developing care and treatment, and service users' responsibility for involvement in their own care.

Approach: A conceptual discussion of the literature is presented which leads to the suggested development of a new model of care to underpin the implementation of mental health practice.

Findings: The biomedical model of mental health predominates explanations of the aetiology of disease, the development of treatments and the delivery of care. Other models such as the bio-psycho-social model and the spiritual model have also been developed. Moreover, in their practice, professionals often adhere to the traditional model that underpins their professional training.

Originality: A user-led model of care to frame the delivery and implementation of mental health support is posited and it is suggested that professionals need to receive training which reinforces developmental rather than routinised learning. Such training would enable them to respond to ways of innovative working which reinforce the autonomy and power of service users in their experience of mental health care and support. This suggests the potential of both a new way for professionals to work alongside service users, recognising the primacy of their expertise, and puts forward a novel emphasis on the kind of training practitioners receive.

My Reflection as an Expert-By-Experience

My brain is a mind-brain. My brain is a biological brain. I experience a separation between the mind as the seat of memory and thought and the brain as a biological set of changing chemicals. How can my mind be reduced to chemicals? Where does it place the human that I am and the emotions and feeling and memories that are made up of me? In some ways it is easier to experience the brain as a set of chemicals reacting wrongly in the context of psychosis; however, this reduces my belief in my spirituality and my uniqueness as a person. Who or what am I? Am I condensed to a set of chemical reactions or is my lived experience a spiritual and meaningful experience? What is easier to believe? If psychosis is a spiritual experience, am I being punished by God? Or as a chemical imbalance, what is it to be human? Is a human a collection of synapse networks, or a person who is more than that?
\end{abstract}

\section{Introduction}

Professional decisions and the choices about the clinical interventions that are offered to people who experience mental illhealth are often founded on application of the biomedical model of mental health; this determines how people are treated, what interventions they receive and how efficacy of different treatment choices is assessed [1]. Moreover, research into the development of new interventions is very often based on a neuro-psychiatric understanding of the manifestation of mental ill-health derived from evidence of randomised control trials (RCTs) and systematic reviews [2,3]; such models reject the validity of user knowledge [2]. For example, recently, the Earlham Institute (22.01.2021) reported a collaboration in developing a new drug for schizophrenia, citing this condition as a neuropsychiatric condition that could be ameliorated by effective drug therapy. Additionally, very often, application of the biomedical model in mental health care dictates the use of drug treatments in managing the symptoms of mental illness, rather than the potential of other treatment methods, such as talking therapies. Thus, implementing the biomedical model of 
mental health has many implications for the user experience of mental health care underpinning the way care is delivered, how interventions are provided, and the treatment choices put in place.

In this article I discuss the application of the biomedical model to mental health care [1] and rehearse well-founded arguments that the use of this framework reduces the lived experiences of mental distress to complex neuro-psychiatric processes which are only understood in terms of biological functioning [4]. Moreover, I posit that research about treatment efficacy [2,3], derived from the biomedical model of care, devalues the position of expert lived experience. I argue the case as an expert-by-experience and academic [5,6], for a move towards a user-led model of care, in which service users own their own knowledge and identity and reclaim the power that is taken away from them. I argue for the validity of user knowledge and a user model of mental health based on value and respect for the user experience, for their expertise-byexperience.

I wrote this article out of a complex reaction to the Cartesian dualism of the mind and body split that comes from Western thought. For those of us with mental health issues our understanding of our lived experiences of mental health are central to our understanding of who we are and what it is to be human. The opening reflection serves as an introduction to this article and underlines the complexity and importance of these arguments to those with lived experience. Fook [7] reinforces the need to reflect on knowledge and how this is key to understanding our relationship with the wider context of theory that surrounds our knowledge base as professionals, service users, academics, and researchers.

\section{My Reflection as an Expert-by-Experience}

My brain is a mind-brain. My brain is a biological brain. I experience a separation between the mind as the seat of memory and thought and the brain as a biological set of changing chemicals. How can my mind be reduced to chemicals? Where does it place the human that I am and the emotions and feeling and memories that are made up of me? In some ways it is easier to experience the brain as a set of chemicals reacting wrongly in the context of psychosis; however, this reduces my belief in my spirituality and my uniqueness as a person. Who or what am I? Am I condensed to a set of chemical reactions or is my lived experience a spiritual and meaningful experience? What is easier to believe? If psychosis is a spiritual experience, am I being punished by God? Or as a chemical imbalance, what is it to be human? Is a human a collection of synapse networks, or a person who is more than that?

\section{Background}

\section{The Biomedical Model of Mental Health}

The evidence-base for the effective development and delivery of medical interventions for people who experience mental ill health is often founded on underlying assumptions of the biomedical approach of care [1]. In the development of clinical interventions, levels of evidence (sometimes called hierarchy of evidence) are assigned to studies based on the methodological quality of their design, validity, and applicability to patient care [2,3]. Level 1 evidence is drawn from systematic reviews of RCTs (randomised control trials) or three or more RCTs of good quality that have similar results. Whereas service user and carer knowledge in the hierarchy of evidence-based treatments are located at level VII alongside evidence from the opinion of authorities and/or reports of expert committees [2]. This reduces the status of lived experience to the level of opinion and belief, devaluing the position of lived experience in the research evidence hierarchy [4].

Moreover, the evaluation framework for the efficacy of treatment methods does not apply only to drug treatment therapies; for example, drawing on the 'roadmap for mental health research in Europe initiative (ROAMER) work package 5', Wittchen et al. [8] consider that an improved future research agenda for psychological therapies in mental health should embrace a "Science of Behaviour". Thus, despite psychological approaches drawing on the social sciences and more social forms of research, Wittchen et al. [8] advocate that research into the effectiveness of psychological interventions should be founded on the biomedical model. This highlights the establishment's commitment to application of the biomedical model to the development, delivery, and implementation of clinical interventions in mental health.

Kinderman and Tai [9] have argued that the application of the biomedical model has been used to justify the use of compulsory treatment for people who experience mental healthcare under the Mental Health Act (MHA) (1983 as amended in 2007) and the Mental Capacity Act (MCA) (2005) in the UK. According to the provision of the Human Rights Act (HRA) (1998) people in the UK, (and more widely in the European Union, through the enactment of the European Convention of Human Rights), have a right to liberty (Article 5) and a right to a private and family life (Article 8). However, the law explicitly allows for exemptions to certain articles in the case of persons 'of unsound mind', under the Mental Capacity Act (2005) and the MHA (1983). Moreover, Article 3 of the Human Rights Act (HRA) (1998) states that 'no one shall be subjected to torture or to inhuman or degrading treatment or punishment'. Despite these rights, many service users believe that their human rights have been set aside [9], and, in actuality, they have perceived mental health care to be degrading and inhumane $[10,11]$ as care is often implemented in adherence to treatments derived from the biomedical approach $[1,31]$ without reference to other treatment models.

Thus, despite a clear focus on the validity of the of neuropsychiatric model to both research and evidence-based practice 
in the development and delivery of mental health interventions [1], Deacon [12] has noted that the worsening chronicity and severity of mental disorders reveals a mental health crisis against which the biomedical paradigm 'has proven ineffectual'. Moreover, there must be an honest debate [12] about the effectiveness of the biomedical model to address a mental health crisis, which has not been ameliorated by the widespread use of psychotropic medication and promotion of the 'biologically-based brain disease'. This suggests the limitations of the biomedical model, which has led to the development of calls for the acknowledgement of the validity of other forms of knowledge besides those advocated by the biomedical model, and points to the development of new ways of understanding mental health difficulties.

\section{The Place of Other Models of Mental Health}

The bio-psycho-social model was developed by Engel [13] nearly 50 years ago who posited that three elements contribute to the development of mental ill-health: the biological, psychological, and social factors. Drawing on this discussion, Kinderman [14] was one of the first authors to develop a psychological model of mental disorder based on the disturbance or disruption of psychological processes. Living in poverty can generate feelings of disillusionment, hopelessness, and learned helplessness, which can lead to depression; such a relationship between cause and effect confirms the evidence for a psychological cause to depression. Furthermore, in later work, Kinderman et al. [15] undertook research to identify the link between family heritability and the development of mental illness. The authors found that rather than hereditary biomedical markers causing intergenerational experiences of mental ill-health, it was the life events (childhood abuse and bullying, and stressful life events in adulthood) which were the strongest direct predictors of mental health problems (depression and anxiety). Thus, Kinderman et al. [15] concluded that a combination of biological, social, and circumstantial factors led to the transmission of mental health issues across families.

Moreover, the unequal distribution of the social determinants of health can also contribute to the development of poor mental and physical health as described by Marmot [16], and in his review 10 years later [17]. Disadvantages, such as adverse childhood events, being a victim of abuse, poor housing, poverty, traumatic events, and poor working conditions, can impact on a child at birth, and the influences of such negative factors can accumulate over time throughout their life course [16-19]. Thus, children facing multiple risks experience an increased chance of sustained childhood and later life mental health difficulties, although the mechanisms by which this happens can be complex and inter-related [19]. The biomedical model is inadequate in explaining such causes [18]; moreover Thachuk [4] argues that the application of the biomedical model pathologises people who experience mental ill-health because it prevents them from challenging the socio-political norms that perpetuate poverty and mental ill health with its emphasis on the cause of mental ill-health as a physical disease.

Thus far, the impact of biomedical, psychological, and social circumstances on the development of poor mental health has been explored, as indicated in the bio-psycho-social model of mental health. Over recent years, attention has also been paid to other factors, alongside these three, which also contribute to the development of poor mental health, such as the recognition of cultural and spiritual dimensions which are an essential expression of good mental health. Saad et al. [20] argue the importance of a spiritual dimension to models of mental health highlighting that the application of this novel view of the human being would 'bring remarkable transformations to the concepts of health, disease, treatment, and cure'. This highlights how a radical transformation in the conceptualisation of mental health can bring about dramatic change in the care and treatment offered to service users.

Furthermore Babalola et al. [21] have noted how the traditional Cartesian dualism has dominated the mind-body split in mental and physical health posited in Western thinking and reject the division between the physical and the mental. Babalola et al. [21] highlight that there are many different and distinct explanatory models for the experiences of mental distress in different cultures and advocate that it is important to understand how people from diverse cultures and ethnic backgrounds respond to mental ill-health. These models not only indicate different explanations for the understanding of health, pathology, and normality, but also point at different ideas about what constitutes personhood. This thus highlights the diverse ways in which different cultures respond to and understand the concept of mental health. Moreover, compulsory treatment under the auspices of the MHA (1983 amended 2007) is more often given to people from BAME (Black, Asian, Minority Ethnic) backgrounds than from the White majority population and mental health services may be open to criticisms of institutional racism [22-24]; this is in part due to differing experiences and understanding of the aetiology of mental ill-health.

Thus, different models, in addition to the biomedical model of mental health, have been developed by professionals from different backgrounds. The limitations acknowledged in these models, developed primarily by academics, researchers, and practitioners, leave a gap for the proposal of a new framework of care based on innovative practice, research and training which starts to acknowledge the role of the user expert in contributing to these areas. Thus, in the next section, I propose a user-led model of mental health, which draws on the validity of expertise-by-experience.

\section{The Proposed User Led Model of Mental Health}

The user led model of mental health proposed in this article reinforces the recognition of the strengths and abilities of service users, emphasising their expertise-by-experience. This model is 
focused on the centrality of user expertise at the center of care. It highlights the agency and autonomy of users, acknowledging their strengths in fighting the debilitating effects of mental distress. In this proposed model, the validity of user led knowledge is not only recognised through individual knowledge but rather through the collective knowledge of user communities [25]. Group activism recognised in the user movements of the 1980s, such as Gay Pride and Mad Pride, embraces the importance of the collective knowledge derived from mutual aid, support, and group identity [26].

Thus, the user led model, proposed in this paper, draws on examples of innovative user involvement in interventions, practice, and research; this focus illustrates the potential validity of this model as it expands across all elements of mental health care. Recent innovations in practice such as shared decision-making in mental health care [27], highlight that each partner is equally responsible for clinical decisions in the exchange between the prescriber and service user [28]. The institution of personal budgets and direct payments in service provision, in which the service user is provided with cash payments to arrange their own care following an assessment, underlines recognition of their ability to choose and direct their own care, as detailed in The Care Act [29]. Novel developments of user led care embodied in the Hearing Voices Network and groups that emphasise service users' own strategies in managing voice hearing are underpinned by respect for service user knowledge [30]. Furthermore, the institution of peer support workers in mental health services reinforces the importance of experiential knowledge in recovery, confirming the importance of expertise by experience in formal mental health care provision [31]. Such innovations, which underline user control in treatment choices and interventions, underpin the development of this model, rejecting the approach exemplified in the biomedical model which emphasises the primacy of the psychiatrist's wisdom and of the mental health team in clinical decision-making.

Furthermore, the involvement of service users in research $[32,33]$ rejects the sole validity of the biomedical model that reinforces the place of clinical studies in determining the success of new drug treatments [3]. The involvement of experts-by-experience in co-producing training for social work students [34,35], health professionals and even of psychiatrists emphasises the validity of experiential knowledge in supporting the professional development of mental health staff. This highlights the relevance of user experience to developing the future professional workforce.

Common factors that define the user led model are found in the examples of innovative practice described above. The development and implementation of these examples of practice redress power imbalances between professionals, researchers, academics, and service users acknowledging the validity of users' rights, their viewpoints, and their control over choices in their lives [27,28]. Moreover, the examples reinforce the validity of knowledge derived from user expertise based on experiential wisdom, recognising their ability and capacity to contribute in an equal way to research, training or choices about their medication, treatment interventions or lifestyle. This sense of agency and autonomy is a central factor in the proposed service user led model. In the following section, the potential influence of this proposed model on professional practice is discussed as the impact of models of care on the wider implementation of support are explored.

\section{The Influence of Models in Mental Health Care}

The model of mental health used to underpin practice is of key importance to the implementation of professional interventions. It highlights certain assumptions about the way mental ill health should be treated, the interventions that should be initiated, and, also, the aetiology of disease. The proposal of the user-led model in this article overlaps to some extent with the recovery model, which emerged in the last 30 years, and has been perceived as a service user led model of care.

The recovery model [36] views mental illness from a perspective radically different to traditional psychiatric approaches. Important elements to recovery are [37] identity, the service provision agenda, the social domain, power, and control, hope and optimism, risk, and responsibility. Moreover, recognition of the importance of taking part in social activities such as education, training, volunteering and employment opportunities has been perceived as important in underpinning the process of individual recovery. Jacob [37] noted how the vibrant user movement in the west argued for different perspectives and approaches to the biomedical model, however Morin and Franck [38] have argued that recovery from mental illness has lost its user focus. Recovery can now be defined in two incompatible ways [38]: on the one hand, service users define recovery as the attainment of a meaningful and valued life, rather than the absence of symptoms; while on the other, psychiatrists have developed a 'medical' model of recovery placing the emphasis on elimination of symptoms and return to normal functioning. Such a dichotomy reduces the validity of the recovery model as a service user-led model and suggests it is now at risk of becoming appropriated by services and professionals. This suggests the need for the development of a new user-led model of care based on innovations in practice, research, and policy.

Kinderman [14] reminds us that different professional groups employ different models to inform their practice. For example, the social worker may support a service user to address difficult social circumstances s/he may experience using a social perspective to underpin his/her work (39), whilst the psychiatrist may prescribe medication to manage any underlying biological disfunction or the occupational therapist may support the service user to manage day- 
to-day tasks using an occupational model. Thus, different models inform the practice of each professional group which has its own professional identity and training.

However, the frameworks which govern the models used by professionals are not always firmly demarcated; King et al. [40] explored in their research whether professionals adhered to the specific model that they reported they worked to, or, in practice, used another framework to guide their interventions. The study concluded that respondents did not consistently use the same theoretical model, but adopted a framework depending upon the context they found themselves in and on the issue that the service user presented. This finding evidenced that clinicians in this study either took a flexible approach to each individual service user or shifted their theoretical beliefs when faced with a practical dilemma; possibly suggesting that professionals revert to traditional models of care when working in practice.

This suggests the need for person-centred care underpinning models of practice to enable professionals to empower and support service users. Ramage et al. [41] have argued for a form of compassion-based care building on the importance of a framework of relationship-based care. Empathy and compassion can lead to a change in the structure of the brain ref and can promote trust [41], which can improve the building of attachments in service users. Stable attachments enable personal growth and change. Selfcompassion can impact positively on physiological and emotional wellbeing [41], thus evidencing that relational mental health models which promote self-compassion can have a positive impact on the mental health care of clients.

Such a model has implications for the care and support of service users who experience mental distress, placing them at the centre of decision-making and highlighting their individual needs; a mode of practice reflected in the innovative examples of intervention highlighted in the proposed user-led model of care. For example, shared decision-making in mental health care is based on a relationship founded on respect and value for the individual [28]; implementation of HVN support is established on a belief that the individual is best placed to develop their own self-management strategies [30]; whilst direct payments in place of arranged care are founded on trust in the individual to manage and direct their own care needs [29]. Thus, the user led model of care resounds with innovative person-centred practice which places compassion and the therapeutic relationship at the centre of support [41]. Furthermore, the user-led model of care has implications for the professional development of practitioners and clinicians, as they adapt and develop their practice, as is further discussed below.

\section{The Learning Needs of Professionals}

The impact of the different models used by professionals and the implementation of the user led model of care suggests the need for tailored training to be made available to practitioners and clinicians to enable them to develop person-centred care based on respect for the validity of user expertise. King et al.'s [40] study suggests that professionals may revert to more traditional patterns of practice acting in a routinised way towards their practice, rather than acquiring and synthesising new knowledge to innovate their approaches [42].

Avby et al. [42] explored the use of knowledge and learning in childcare practice in Sweden, they distinguished between adaptive and developmental modes of learning. The former can be understood as a process through which a person acquires the capacity and skills needed to routinely handle and master certain tasks or situations; whereas the latter occurs when individuals or groups challenge routinised forms of learning and knowledge to develop new ways of managing complex problems involved in a certain task or job. Developmental learning occurs when new knowledge is subsumed into professional practice to generate new ways of working. It appears that many professionals often revert to more traditional ways of practice rather than embracing novel approaches to mental health care.

This underlines the importance of reinforcing information about new ways of working which encourage professionals to adopt alternative frameworks to those traditionally used. Moreover, if professionals begin to recognise and understand the diverse models that inform the causes and aetiology of illness, this will enable them to exhibit many skills and be conversant in the delivery of treatment from different models. For example, psychiatrists will have to give attention to both the psycho-social as well as the bio-medical [43]. The proposed new user -ed model of care emphasises the strengths of service users and recognises the validity of their knowledge, highlighting the importance of moving from a professional-centred explanation of mental ill-health to that advocated by service users themselves.

\section{Conclusion}

The biomedical model [1] currently dominates the explanation for the aetiology of disease, and its practical application determines the kinds of treatments which are offered in mental health care. Other new models of mental health care have also been developed since the emergence of the biomedical model, such as the biopsycho-social model [13], the psychological models [14] and the spiritual model of care [20]. As my opening reflections show, this topic is of key importance to the lives of many people with lived experience and is more than a theoretical construct about the evidence that informs the models which influence professional practice.

The new model proposed in this article underlines a focus 
on a user-defined framework for care, underpinned by an acknowledgement of the validity of both individual and collective experiential knowledge. Initiatives which emphasise service users' control and power over treatment choices, their right to choose, and the rebalancing of differential power between service user and professional are key to understanding this model. It encompasses notions of inclusion, empowerment, and involvement - recognising service users as experts-by-experience with a real stake in the delivery of their own care, and the wider context of the development and implementation of services.

Professionals need to foster a developmental approach to learning, rather than adaptive approach [42] enabling them to challenge their routinised practices and to enhance their understanding of new forms of practice, to encourage them to innovate. This requires the implementation of training processes which reinforce new learning rather than the traditional default model of professional groups [40]. The user-led model of care links to many empowering practices such as SDM [27], involvement of service users in research [32,33], in co-producing training for health and social care professionals [34,35]. This emphasises a new perspective which is founded on respect for expertise-byexperience and acknowledgement of the validity of experiential knowledge, key to moving forward to improving the mental health of people who experience mental distress.

\section{References}

1. Tekkalaki B, Tripathi A, Trivedi J (2014) Welcome biological breakthroughs, supply psychosocial insights. Mens Sana Monogr 12(1): 79-91.

2. Ackley B, Swan B, Ladwig G, Tucker S (2008) Evidence-based nursing care guidelines. Medical-surgical interventions, St. Louis, MO: Mosby Elsevier, USA.

3. Earlham Institute (2021) Investigating new treatment for schizophrenia as first Psychiatry Consortium funded project announced. UK.

4. Thatchuk A (2011) Stigma and the politics of biomedical models of mental illness. International Journal of Feminist Approaches to Bioethics 4(1): 140-163.

5. Fox J (2016) Being a service user and a social work academic: balancing expert identities. Social Work Education 35(8): 960 - 969.

6. Fox J (2017) The Contribution of experiential wisdom to the development of the mental health professional discourse. Schizophr Bull 43(3): 481485.

7. Fook J (2014) Learning from and researching (my own) experience: A critical reflection on the experience of social difference. Witkin S, et al. (Ed.) Narrating Social Work through Autoethnography, New York, USA. pp. 120-140.

8. Wittchen H, Knappe S, Schumann G (2014) The psychological perspective on mental health and mental disorder research: introduction to the ROAMER work package 5 consensus document. International Journal of Methods in Psychiatric Research Int. J. Methods Psychiatr Res 23(Suppl 1): $15-27$.

9. Kinderman P, Tai S (2008) Psychological models of mental disorder, human rights, and compulsory mental health care in the community. Int
J Law Psychiatry 31: 479-486.

10. Sainsbury Centre for Mental Health (2006) Choice in mental health: briefing paper 31, London: Sainsbury Centre for Mental Health.

11. Mind (2004) Ward Watch: Mind's report on hospital conditions for mental health patients, London: Mind, England.

12. Deacon B (2013) The biomedical model of mental disorder: a critical analysis of its validity, utility, and effects on psychotherapy research. Clin Psychol Rev 33: 846-861.

13. Engel G (1977) The need for a new medical model: A challenge for biomedicine. Science 196(4286): 129-136.

14. Kinderman P (2005) A psychological model of mental disorder. Harvard Review of Psychiatry 13(4): 206-217.

15. Kinderman P, Schwannauer M, Pontin E, Tai S (2013) Psychological processes mediate the impact of familial risk, social circumstances and life events on mental health. PLoS One 8(10): e76564.

16. Marmot M (2010) Fair Society Health Lives, London: The Institute of Health Equity.

17. Marmot M, Allen J, Boyce T, Goldblatt P, Morrison J, et al. (2020) Marmot Review 10 years old. London, Institute of Health Equity.

18. Tripathi A, Das A, Kar S (2019) Biopsychosocial model in contemporary psychiatry: current validity and future prospects. Indian Journal of Psychological Medicine 41(6): 582-585.

19. Public Health England (PHE) 2018 Guidance Health matters: reducing health inequalities in mental illness.

20. Saad M, de Medeiros R, Mosini A (2017) Are we ready for a true biopsychosocial-spiritual model? The many meanings of spiritual. Medicines 4(4): 79-85.

21. Babalola E, Noel P and White R (2017) The biopsychosocial approach and global mental health: Synergies and opportunities", Indian J Soc Psychiatry 33(4): 291-6.

22. Keating F, Mc Culloch A, Francis E (2002) Breaking the circles of fear: a review of the relationship between mental health services and African and Caribbean communities, London: Sainsbury Centre for Mental Health.

23. Bhui K, Nazroo J, Francis J, Halvorsrud K, Rhodes J, et al. (2018) The impact of racism on mental health. Synergi, UK.

24. Wallace S, Nazroo J, Becares L (2016) Culmulative effect of racial discrimination on the mental health of ethnic minorities in the United Kingdom. American Journal of Public Health 106(7): 1294-1300.

25. Rüsch N, Corrigan P, Wassel A, Michaels P, Olschewski M, et al. (2009) A stress-coping model of mental illness stigma: I. Predictors of cognitive stress appraisal. Schizophr Res 110(1-3): 59-64.

26. Backwith D (2015) Social Work, Poverty and Social Exclusion, Maidenhead: Open University Press/Mc Graw-Hill, USA.

27. Fox J (2021) Shared decision-making: an autoethnography about service user perspectives in making choices about mental health care and treatment. Front Psychiatry.

28. Ramon S, Brooks H, Rae S, O Sullivan M (2017) Key issues in the process of implementing shared decision making (DM) in mental health practice. Mental Health Review Journal 22(3): 257-274.

29. The Care Act (2014) London: HMSO, England.

30. Hearing Voices Network (HVN) nd.

31. Repper J, Aldridge B, Gilfoyle S, Gillard S, Perkins R, et al. (2013) Peer Support Workers: a practical guide to implementation. ImROC, Centre for Mental Health, Mental Health Network NHS Foundation. 
32. Lambert N, Carr S (2018) Outside the original remit': co-production in UK mental health research, lessons from the field. Int J Ment Health Nurs 27(4): 1273-1281.

33. Mc Cauley C, McKenn H, Keeney S, Mc Laughlin D (2017) Service user engagement: a co-created interview schedule exploring mental health recovery in young adults. J Adv Nurs 17(73): 2361-2372.

34. Hatton K (2017) A critical examination of the knowledge contribution service user and carer involvement brings to social work education. Social Work Education the International Journal 36(2): 154-171.

35. Fox J (2020) Perspectives of experts-by-experience: an exploration of lived experience involvement in social work education, Social Work Education the International Journal pp. 1-18.

36. Leamy M, Bird V, Le Boutillier C, Williams J, Slade M, et al. (2011) Conceptual framework for personal recovery in mental health: systematic review and narrative synthesis. British Journal of Psychiatry 199(6): 445-452.

37. Jacob K (2015) Recovery model of mental illness: a complementary approach to psychiatric care. Indian J Psychol Med 37(2): 117-119.
38. Morin L, Franck N (2017) Rehabilitation interventions to promote recovery from Schizophrenia: a systematic review. Front Psychiatry 8: 100 .

39. Tew J (2011) Social Approaches to Mental Distress. London: Macmillan; Red Globe Press,USA

40. King C, Fulford, B Williamson T, Dhillon K, Vasiliou-Theodore C, et al. (2009) Model values: race values and models in mental health, London: University of Warwick and Mental Health Foundation.

41. Ramage D, Ellis S, Marks-Maran D (2018) The Tidal model in mental health practice: a person-centred approach. J Psychiatr Ment Health Nur $7(3): 137-143$.

42. Avby G, Nilsen P and Ellström P (2017) Knowledge use and learning in everyday social work practice: a study in child investigation work. Child and Family Social Work 22: 51-61.

43. Frost B, Tirupati S, Johnston S, Turrell M, Lewin T, et al. (2017) An integrated recovery-oriented model (IRM) for mental health services: evolution and challenges. BMC Psychiatry 17: 22 - 39. 